\title{
Adaptive Evolution Coupled with Retrotransposon Exaptation Allowed for the Generation of a Human-Protein-Specific Coding Gene That Promotes Cancer Cell Proliferation and Metastasis in Both Haematological Malignancies and Solid Tumours: The Extraordinary Case of MYEOV Gene
}

\author{
Spyros I. Papamichos, Dimitrios Margaritis, and Ioannis Kotsianidis \\ Department of Haematology, School of Medicine, Democritus University of Thrace, 68100 Alexandroupolis, Greece
}

Correspondence should be addressed to Spyros I. Papamichos; spmedauth@yahoo.gr

Received 15 June 2015; Accepted 27 September 2015

Academic Editor: Ben J. Mans

Copyright (C) 2015 Spyros I. Papamichos et al. This is an open access article distributed under the Creative Commons Attribution License, which permits unrestricted use, distribution, and reproduction in any medium, provided the original work is properly cited.

\begin{abstract}
The incidence of cancer in human is high as compared to chimpanzee. However previous analysis has documented that numerous human cancer-related genes are highly conserved in chimpanzee. Till date whether human genome includes species-specific cancerrelated genes that could potentially contribute to a higher cancer susceptibility remains obscure. This study focuses on $M Y E O V$, an oncogene encoding for two protein isoforms, reported as causally involved in promoting cancer cell proliferation and metastasis in both haematological malignancies and solid tumours. First we document, via stringent in silico analysis, that MYEOV arose de novo in Catarrhini. We show that MYEOV short-isoform start codon was evolutionarily acquired after Catarrhini/Platyrrhini divergence. Throughout the course of Catarrhini evolution MYEOV acquired a gradually elongated translatable open reading frame (ORF), a gradually shortened translation-regulatory upstream ORF, and alternatively spliced mRNA variants. A point mutation introduced in human allowed for the acquisition of MYEOV long-isoform start codon. Second, we demonstrate the precious impact of exonized transposable elements on the creation of MYEOV gene structure. Third, we highlight that the initial part of MYEOV long-isoform coding DNA sequence was under positive selection pressure during Catarrhini evolution. MYEOV represents a Primate Orphan Gene that acquired, via ORF expansion, a human-protein-specific coding potential.
\end{abstract}

Spyros I. Papamichos dedicates this writing to Thomas I. Karkalis.

\section{Introduction}

It has formerly been suggested that at least some of human (Homo sapiens) major diseases could partially likely be related to genetic maladaptations during the recent evolutionary past [1]. The incidence of cancer in human is rather high as compared to chimpanzee (Pan troglodytes) [2]. However, previous analysis [3] has shown that numerous human cancerrelated genes are highly conserved in chimpanzee, containing intact open reading frames (ORFs). Only minor differences were reported between species [3]. Therefore whether human genome includes species-specific cancer-related genes or gene isoforms that could potentially contribute to a higher cancer susceptibility remains yet obscure.

Of note, MYEOV (also known as OCIM, National Center for Biotechnology Information [NCBI] Gene ID: 26579) was not included in the human cancer gene data set used in the analysis by Puente et al. [3]. MYEOV is a noncensus cancer gene that during the last 15 years has been reported as causally involved in promoting cancer cell proliferation and metastasis in both haematological malignancies and solid tumours [4-11]. 
The gene has the potential to generate via alternative splicing six mRNA variants encoding for two protein isoforms $[6,9,12]$, namely, for a 313-amino-acid (aa) peptide (MYEOV-313) as well as for a shorter 255-aa peptide (MYEOV-255). Solid Western-blot assays support the production of both the proteins $[9,12]$ while MYEOV313 expression has been associated with poor prognosis in patients with multiple myeloma [9]. Both MYEOV-313 and MYEOV-255 seem to be directed to the membrane [6] but are of, yet, unknown biological function.

MYEOV has been shown to be epigenetically regulated via a DNA-methylation mechanism [5] while at the protein synthesis level the gene seems to be tightly controlled by upstream ORFs (uORFs) [12]. Importantly, MYEOV second pseudosignal generates a long, weak Kozak signal, uORF that could significantly impair translational efficiency [13-15].

In 2012, MYEOV was characterized as a "Class I" hominoid-specific de novo protein-coding gene [16], id est, deduced to encode for protein exclusively in human. Xie et al. [16] identified an ORF disrupting mutation that was present in MYEOV syntenic locus in multiple higher primates, ranging from chimpanzee to rhesus monkey (Macaca mulatta). Intriguingly, the "shared disabler" [17] located in MYEOV313 start codon [16]. Accordingly, the authors [16] excluded $M Y E O V$ origination via other molecular mechanisms known to generate novel genes [18] and annotated MYEOV as a human-specific de novo protein-coding gene, eventually concluding that it is likely de novo origination rather than ORF expansion that drove the origination of hominoidspecific genes [16], such as MYEOV.

Transposable elements (TEs) are known to exert a broad evolutionary impact, promoting genomic plasticity and eventually biological diversity $[19,20]$. Exonized TEs have the potential to induce significant changes in functional noncoding regions of genes while, in extraordinary cases, some could even acquire a novel role as protein-coding modules [21-24], in a biological process called "exaptation" [25]. Interestingly, it was relatively recently documented that TEs could preciously contribute in de novo gene-origination [26-29].

In this paper, we argue about MYEOV representing a human-specific de novo protein-coding gene. We show, via in silico analysis, that MYEOV arose de novo in Catarrhini and that it was adaptive evolution coupled with TE exaptation that eventually allowed for the generation of a human-specific primary ORF.

\section{Materials and Methods}

2.1. Inquiring into MYEOV Origin and Validating MYEOV Orthologous Sequences. MYEOV locus syntenic alignments of numerous vertebrates were extracted from the "Multiz Alignments of 100 Vertebrates" track of the UCSC Genome Browser Database [30-33].

BLASTN [34] search against the GenBank human genomic database and BLASTP [34] search against the GenBank nonredundant protein database were used to exclude $M Y E O V$ origination via gene duplication and to verify unambiguous MYEOV orthologs; the stringent filters described previously were applied $[16,35]$.
MYEOV orthologs annotated in the respective databases NCBI, Ensembl, and OrthoDB [36] were identified by searching with the gene name. Whole-genome shotgun (WGS) sequence contigs, including within the DNA segments used to curate the reference genomic sequences of $M Y E O V$ orthologs, were downloaded from the NCBI Gene database (http://www.ncbi.nlm.nih.gov/gene/). The WGS sequence contigs were used to build MYEOV locus alignment blocks between human and the species with annotated orthologs, via Clustal Omega [37]. Each alignment block was manually scrutinized for validating the primary ORF (pORF) of the annotated orthologs.

To estimate the degree of evolutionary constraint on MYEOV coding DNA sequence during evolution in the Catarrhini genome, the average human-rhesus $K_{a} / K_{s}$ ratio was calculated [38]; MEGA6 software was used in the analysis [39]. Ratios for MYEOV second and third exon coding DNA sequences were calculated separately.

Provided in the study by Finstermeier et al. [40] is a beautiful phylogenetic tree that clarifies the nomenclature and supports the mammalian classification and the estimated divergence ages used in the text.

\subsection{Scanning MYEOV and MYEOV Evolutionary Antecedents} for the Presence of Integrated TEs. MYEOV and MYEOV orthologous genomic sequences were scanned for the presence of TEs by RepeatMasker [41]. The program was run in both default and sensitive mode, using a matrix optimal for MYEOV GC level to avoid false masking. Respective searches using PLOTREP [42], the TranspoGene database [43], and BLASTN $[34,44]$ were also performed.

MYEOV locus automated alignments downloaded from the UCSC Genome Browser Database [30-33] were used to extract syntenic DNA segments in numerous mammals that flank, 500 nucleotides (nts) in the $5^{\prime}$ and the $3^{\prime}$, MYEOV-313 start codon. BLASTN was used to align these DNA segments with corresponding WGS sequence contigs for extracting the original DNA sequence in each species. That is because the "Multiz Alignments of 100 Vertebrates" track of the UCSC Genome Browser Database excludes multiple inserts located between the alignment blocks of the synteny. Each WGS sequence contig was then realigned to human MYEOV. The DNA segments eventually extracted were scanned by RepeatMasker [41] for the presence of integrated TEs.

2.3. MYEOV Upstream ORF and Splice Site Computational Analysis. Search for MYEOV uORFs was performed with the ORF Finder program (http://www.bioinformatics.org/sms2/ orf_find.html). Search parameters were set as previously suggested [45].

MYEOV core splicing signal strength values were accessed with the Human Splicing Finder Version 2.4.1 program [46], as previously described [47].

2.4. Searching for $m R N A$ Expression Evidence in Nonhuman Primates. BLASTN searches with human MYEOV mRNA sequences against the GenBank expressed sequence tags (EST) database were used to identify matching expressed mRNA sequences in nonhuman primates. 
2.5. MYEOV Common Single Nucleotide Polymorphisms and Confirmed Somatic Mutations Analysis. Common single nucleotide polymorphisms (SNPs), located in MYEOV genomic sequence, were extracted from the "Common SNPs" track of the UCSC Genome Browser Database [30-33].

MYEOV confirmed somatic mutations annotated in the catalogue of somatic mutations in cancer (COSMIC) [48] were identified by searching with the gene name.

\section{Results and Discussion}

3.1. From a Eutherian-Mammal Noncoding Sequence to a Human-Protein-Specific Coding Gene. MYEOV BLASTN search against the GenBank human genomic database yields no significant similarity with any coding genomic sequences other than itself.

Syntenic alignments, extracted from the "Multiz Alignments of 100 Vertebrates" track of the UCSC Genome Browser Database [30-33], signify that the DNA segment where $M Y E O V$ locates emerged in eutherian mammals. The DNA segment is absent in all outgroups ranging from marsupials to lamprey. Of note a number of species, for example, Myomorpha, Sciuromorpha, and Lagomorpha, lack a large portion of the syntenic region (Supplementary Figure 1 in Supplementary Material available online at http://dx.doi.org/ $10.1155 / 2015 / 984706)$. This finding could explicate the inability to detect a mouse myeov transcript via zoo blot analyses under low-stringency hybridization conditions, as reported previously [6].

$M Y E O V$ is reported in the Gene database from NCBI (http://www.ncbi.nlm.nih.gov/gene/) to have sixteen orthologous genes, exclusively present in higher primates (Anthropoidea). Fifteen protein-coding genes as well as a pseudoortholog in rhesus monkey are annotated.

MYEOV-313 BLASTP search against the GenBank nonredundant protein database [34] yields however an intriguing finding. The peptide predicted to be encoded from Bolivian squirrel monkey (Saimiri boliviensis) MYEOV ortholog presents higher amino acid coverage with MYEOV-313 protein compared to the respective peptide encoded from chimpanzee MYEOV ortholog, despite Bolivian squirrel monkey being a primate far more distantly related to human than chimpanzee is.

Of note, OrthoDB [36] reports yet another MYEOV protein-coding ortholog in dolphin (Tursiops truncatus), raising the issue of the locus functionality in ancestral genomes to be subsequently lost in multiple lineages and later regained in higher primates [17, 49, 50].

The plausible interpretation of these intriguing findings is that some of the automated annotations of MYEOV orthologous ORFs provided by the NCBI Eukaryotic Genome Annotation Pipeline and the Ensembl Genebuild [51] contain inaccuracies [49]. Elucidated below is the evolutionary path leading to $M Y E O V$.

Interestingly, one of the first functional genetic modules that emerged during MYEOV evolution in the mammalian genome was the long $\mathrm{uORF}$, reported previously to participate in the regulation of the gene's translational efficiency [12]
(Figure 1). The upstream ATG trinucleotide demarcating this long uORF seems fixed in Catarrhini but in depth phylogenetic analysis indicates that it could be of ancestral status (Figure 2). The trinucleotide appears transmuted in Platyrrhini probably due to either emergence of the ACG trinucleotide in a common Platyrrhini ancestor or parallel mutations in these species. With regard to the TGA trinucleotide originally delimiting the long uORF, it appears evolutionarily fixed in higher primates. Eventually the presence of these two trinucleotides in the genomic sequence allowed for the occurrence in Catarrhini of a primal 219 nts long uORF (Figure 2).

Circa (ca.) $46 \mathrm{Ma}$ (Catarrhini/Platyrrhini divergence time) [40], an important mutational event occurred in Catarrhini resulting in the de novo gain of a potentially functional ORF in a genomic region that is noncoding in other primates; that is the acquisition of MYEOV-255 start codon (Figure 2).

Ca. 15.2 Ma (Homininae/Ponginae divergence time) [40] another precious point mutation allowed in Hominines for the shortening of MYEOV long uORF (Figure 2), an event rather meaningful in the context of the gene's translational regulation [45]. Of note, an identical TGA trinucleotide occurring in Bolivian squirrel monkey syntenic region is likely due to a parallel mutation (Figure 2).

Finally, ca. 5.9 Ma (Homo/Pan separation time) [40] a momentous point mutation was introduced in human resulting in the acquisition of MYEOV-313 start codon (Figure 2). An identical trinucleotide appearing in whitetufted-ear marmoset (Callithrix jacchus) syntenic region is due to a parallel mutation and is likely nonfunctional.

Notably, the stop codon delimiting the relatively short pORFs in Old World monkeys (Cercopithecoidea) represents not an ancestral frame disrupting feature that the corresponding hominoid orthologs escaped from since this trinucleotide occurs exclusively in Old World monkeys (Supplementary Data Set 1). In addition it is off-frame with regard to MYEOV coding sequence (Figure 2, Supplementary Data Set 1). That is due to a 14-bp deletion, also fixed exclusively in Old World monkeys, locating short upstream from the stop codon (Figure 2). In the same sense, the stop codon demarcating the pORF in gibbon (Nomascus leucogenys) occurs exclusively in gibbon and orangutan (Pongo abelii). It would feel somewhat more plausible to speculate that parallel mutational events took place in both species rather than assuming the occurrence of a point mutation in a common hominoid ancestor, suffering refutation and evolutionary "refixation" to the antecedent state in succeeding lineages (Figure 2). Of note an orangutan-specific insertion, requiring decidedly additional validation due to shifting very early the respective orangutan MYEOV pORF, translocates out-offrame this $T G A$ trinucleotide (Figure 2, Supplementary Data Set 1).

All the above could signify that MYEOV third exon coding DNA sequence (Figure 2) was not under strong selective constraints during evolution in the Catarrhini genome $[38,52]$. Reinforcing the above, this sequence yields an average human-rhesus $K_{a} / K_{s}$ ratio of 1.14. Accordingly, it was likely neutral drift-directed evolution [53] that drove the $3^{\prime}$ expansion of MYEOV pORF (Figure 2). 


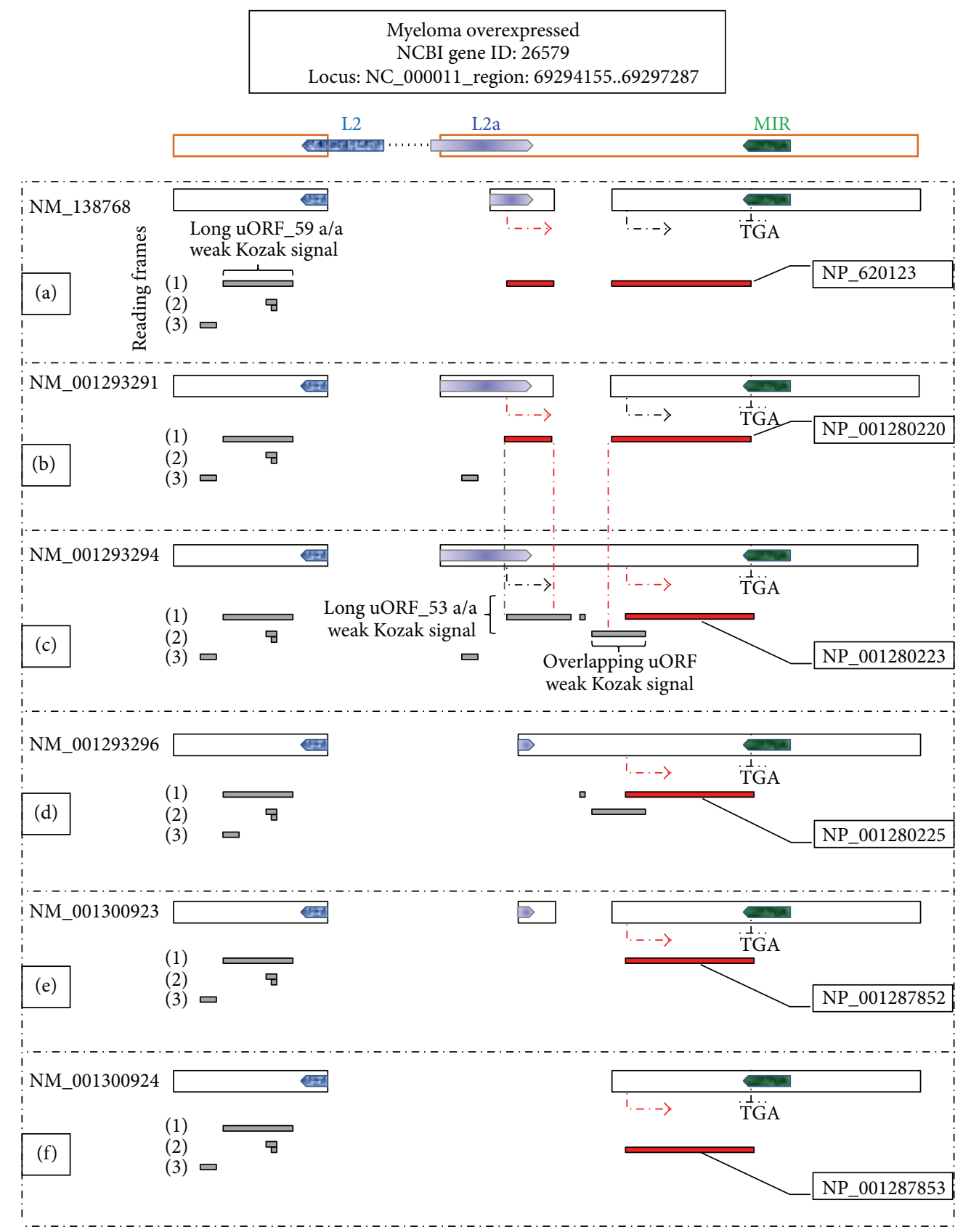

FIGURE 1: MYEOV alternatively spliced variants. Illustrated on top is the genomic organization of MYEOV locus. Bold, orange line boxes correspond to exonic segments of the genomic sequence. Pentagons overlapping the boxes delineate the location and orientation of the three TEs referred to in the text. (a-f) Six MYEOV alternatively spliced variants encode for either MYEOV-313 or MYEOV-255, according to the NCBI Gene database. Bold, black line boxes for MYEOV exonic content corresponding to each variant. Directional arrows correspond to the functional start signals of $M Y E O V$; red directional arrows are for the start codons demarcating the respective pORF in each variant. The common stop codon location (TGA) is also indicated. Provided below the exonic content, depicted as dark-grey horizontal bars and juxtaposed to the respective pORFs (red horizontal bars), are the uORFs present in each mRNA sequence; long uORFs are annotated in the context of significantly impairing translational efficiency. Numbers in parentheses correspond to consecutive reading frames, starting from the first, second, and third nucleotide of each variant. Importantly, a prerequisite for MYEOV long-ORF to occur is the region demarcated by red vertical lines to be spliced out of the mRNA sequence. 


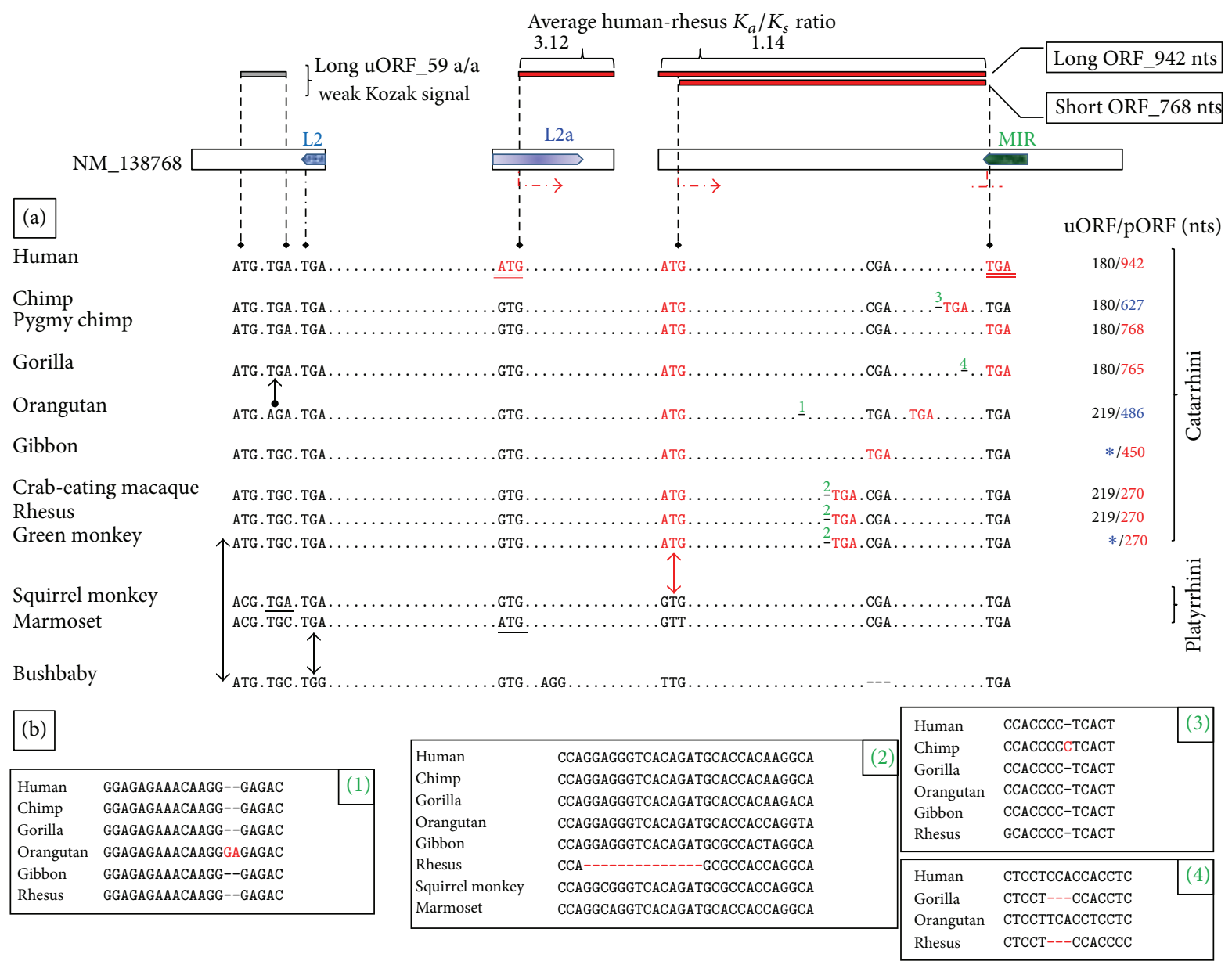

FIGURE 2: From a noncoding sequence to MYEOV. Depicted is MYEOV transcript variant 2 (NM_138768) as described in Figure 1; illustrated are both the translatable ORFs (red horizontal bars) and the long uORF (dark-grey horizontal bar) present in the variant. Shown also are the average human-rhesus $K_{a} / K_{s}$ ratios calculated separately for MYEOV second and third exon coding DNA sequences. (a) Black, discontinuous, vertical lines point to precious trinucleotides of $M Y E O V$ sequence, referred to within the text. Appearing underlined, in red font, are the trinucleotides that demarcate MYEOV-313 coding sequence. Aligned below the human sequence are corresponding trinucleotides from MYEOV syntenic region in 11 primates. Bona fide pORFs in MYEOV orthologs are delimited by respective red font trinucleotides; outof-frame stop codons are presented unaligned. Double-headed red arrow points to the de novo acquisition of MYEOV-255 start codon in Catarrhini. MYEOV-313 start signal is human-specific; an identical ATG trinucleotide appearing in marmoset syntenic region (underlined) is due to a parallel mutation. Double-headed black arrows are for the trinucleotides demarcating the primal 219 nts long uORF that arose in Catarrhini. A black directional arrow points out the shortening of this long uORF in Hominines; an identical TGA trinucleotide occurring in squirrel monkey syntenic region (underlined) is likely due to a parallel mutation. Shown in the right flank is the size of the long uORF/pORF corresponding to each species. The sizes of the pORFs present in chimpanzee and Sumatran orangutan MYEOV orthologs (appearing in blue font) require decidedly additional validation due to the presence of ORF-shifting indels in the respective sequences. Blue asterisks for gibbonand green monkey-specific indels that transmute the respective uORFs. Alignment gaps in bushbaby syntenic region were inserted for clarity. (b) Provided within boxes, numbered in accordance with the superscripts numbers appearing in (a), are syntenic alignments among various higher primates including the indels referred to in the text. With regard to the Cercopithecoidea-specific 14 bp deletion, shown is only one of these species due to space limitations.

On the other hand, MYEOV second exon coding DNA sequence yields an average human-rhesus $K_{a} / K_{s}$ ratio of 3.12. Thus the $5^{\prime}$ expansion of MYEOV pORF during the gene's evolution in the Catarrhini genome, including the acquisition of the human-specific start codon, was likely driven under positive selection pressure [54] (Figure 2).
3.2. Precious Exapted TEs Present in MYEOV Genomic Sequence. MYEOV scan by RepeatMasker reveals the presence of seven TEs in the genomic sequence (Supplementary Data Set 2). Importantly, nonredundant MYEOV modules seem to localize within three of these genetic elements. 
In detail, the constitutively used donor of MYEOV first splicing junction locates in a region reported from RepeatMasker to match an antisense orientation L2 repeat of the long interspersed nuclear element (LINE) class (Figure 1). Of note, this TE provided also the stop codon delimiting MYEOV primal 219 nts long uORF, discussed above (Figure 2).

The stop codon that is common to both MYEOV313 and MYEOV-255 protein isoforms locates in a region reported from RepeatMasker to match an antisense orientation mammalian-wide interspersed repeat (MIR) of the short interspersed nuclear element (SINE) class (Figure 1).

Most importantly the three alternative acceptors of $M Y E O V$ first splicing junction and the initial region of MYEOV-313 coding sequence, including the start codon, are located in a region reported to match a sense orientation LINE L2a repeat (Figure 1). This finding represents a rare exaptation event $[22,55]$ but is somewhat equivocal in the context of the RepeatMasker match appearing highly degenerated and yielding a score relatively close to the Smith-Waterman cutoffs; that is 180 for the ancient MIR, L2, and MER5 sequences [41]. At this point it should be noted however that the accurate annotation of a L2 familyrepeat content and the valid estimation of its original extent are issues very hard to meet since it is not unusual for these ancient, inactive elements to be degenerated beyond recognition $[56,57]$.

A subsequent MYEOV scan using PLOTREP [42] further reinforces that a segment of MYEOV-313 initial coding sequence was likely provided by a TE of the L2 family (Supplementary Data Set 3). The relatively short sequence extracted by PLOTREP, while corresponding to a minor segment of the RepeatMasker match, matches identically to the respective data extracted from the TranspoGene database and to MYEOV DNA segment masked by BLASTN, when a DUST-driven [44] filter for human-specific repeats is applied. Of note, RepeatMasker's results not corresponding well with the respective data extracted from PLOTREP [42], the TranspoGene database [43], and BLASTN represent not an atypical finding; this inconsistency is due to other programs using much smaller databases than RepeatMasker. RepeatMasker's mammalian libraries represent heavily manipulated and expanded versions of the respective Repbase libraries [58].

The above data led us to subsequently inquire into the status of the L2a repeat in MYEOV evolutionary antecedents. Results validated the presence of L2 family-repeat-relics in the corresponding syntenic region in, at least, seven eutherian mammals (Figure 3, Supplementary Data Set 4). Importantly, it is well known that L2s and MIRs underwent active retroposition prior to the placental mammalian radiation $[22,57]$. It is also known that cases of TEs inserted independently in nearby syntenic genomic regions in species identical-bydescent represent extremely rare events with exceedingly low probability to occur [59]. In line with these data, it is highly likely that RepeatMasker not only defining divergently the repeat's size and exact boundaries in each species but also providing different subfamily names for the repeat is due to the high degree of degeneration of the repeat during its evolution in the mammalian genome. Overall, it is particularly plausible that the molecular L2 repeat-fossils, present in the nearby syntenic regions of the seven mammals (Figure 3), were derived from the same $\mathrm{L} 2$ transposable element that was introduced in the common eutherian ancestor.

Another line of evidence to support the above, likely the most conclusive one, would be the probabilistic reconstruction of MYEOV locus ancestral sequence [60]. Indeed, it has been suggested that using RepeatMasker to scan the inferred boreoeutherian ancestor sequence of a given genomic locus would provide more accurate information on the original extent of the ancient TEs included within than running the software in the corresponding human sequence [61], because the ancient repeats would appear much less degenerated in the ancestral sequence. Of note, a series of prerequisites should be met for accurately reconstructing an ancestral sequence [61]. Most importantly, problematic sampling of major lineages and outgroups seems to significantly decrease the accuracy of ancestral sequence reconstruction $[61,62]$. Consequently, Myomorpha, Sciuromorpha, and Lagomorpha lacking a large segment of MYEOV syntenic region likely represent a severe drawback in optimally performing the sampling procedure.

\subsection{MYEOV Full Splicing Potential Was Acquired Relatively} Late during the Gene's Evolution in the Catarrhini Genome. As shown in Figure 4, only one MYEOV canonical splicing signal, id est, allowing for RNA processing by the standard U2 type spliceosome [63], predated the radiation of Haplorhini. Three canonical splicing signals arose in higher primates while two precious standard acceptors appeared later in time.

Importantly the canonical acceptor of MYEOV second splicing junction, namely, of the splicing junction allowing for MYEOV long-ORF to occur (Figure 1), is hominoid-specific (Figure 4).

\subsection{Transcription of MYEOV Locus in Human and Nonhuman} Primates. In human, transcription of $M Y E O V$ genomic locus is driven by a strongly active cryptic promoter sequence locating in MYEOV $5^{\prime}$ untranslated region (5' UTR) [12], between the second and third pseudosignal of the gene (Figure 1). Should this cryptic promoter precede or follow the emergence of the translatable ORF in Catarrhini and be active in other higher primates as well remains to be clarified via subsequent studies, especially because the expressed sequence tags- (EST-) coverage of the genomic sequence in nonhuman primates is low.

Indeed, BLASTN searches with human MYEOV mRNA sequences against the GenBank EST database yield only one, however precious, unambiguous hit (NCBI accession: DC527428). This EST validates transcription of the genomic locus in chimpanzee.

3.5. MYEOV-313 Start Codon Includes Not Common Polymorphic Sites in Human. According to the single nucleotide polymorphism database from NCBI (http://www.ncbi.nlm .nih.gov/snp/), located in human MYEOV gene are 17 common polymorphic sites. None locates in MYEOV-313 start codon. 


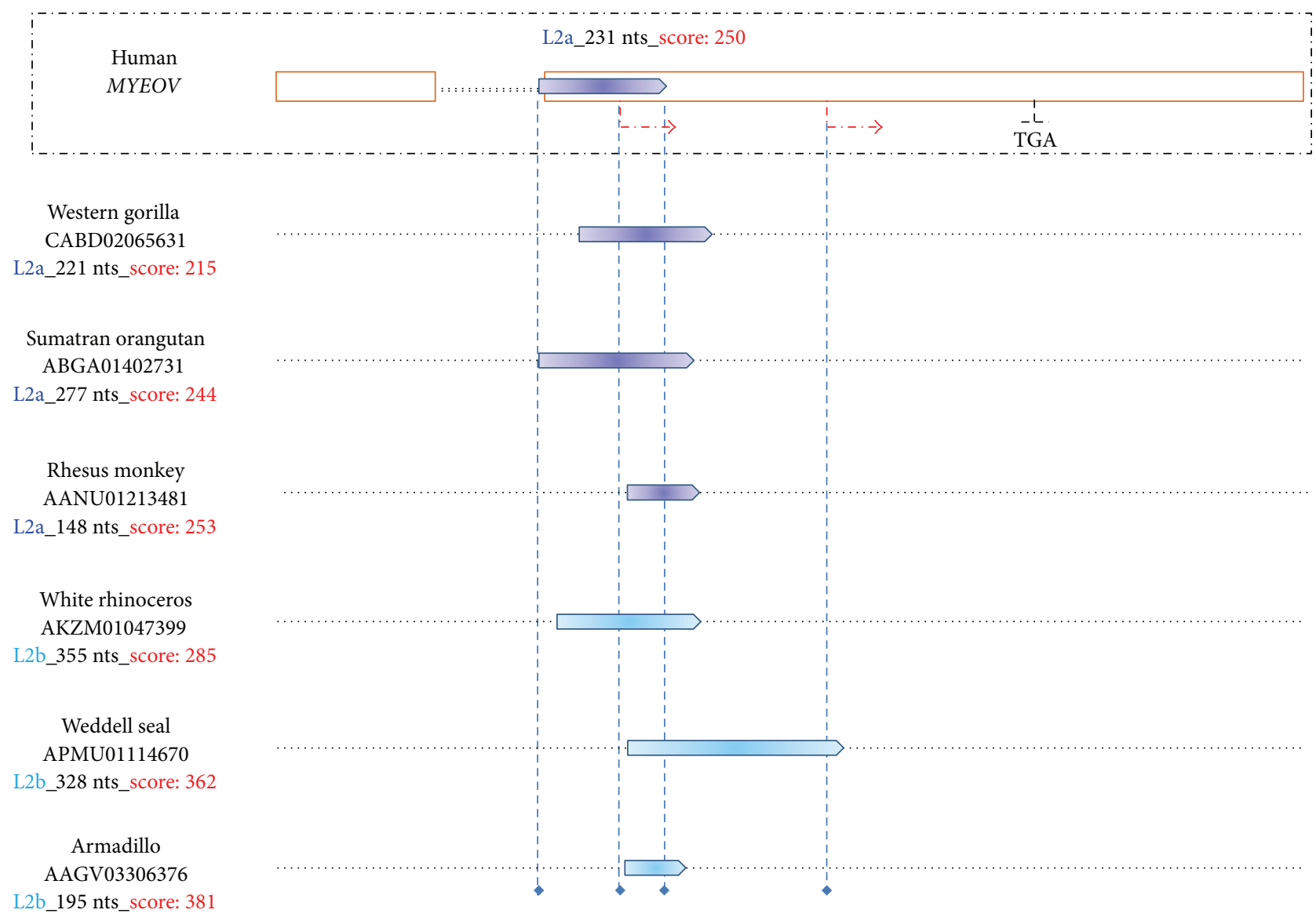

FIGURE 3: Presence of L2 family-repeat-fossils in MYEOV evolutionary antecedents. Upper panel: illustrated on top is $M Y E O V$ locus, as described in Figure 1. Shown is the location of the sense orientation L2 family-repeat followed by RepeatMasker's annotation in the context of the subfamily name appearing in consensus annotation evidence (dark blue font), the size (black font), and the Smith-Waterman score (red font) of the match. Lower panel: depicted is the presence of L2 family-repeat-relics (pentagons) in MYEOV syntenic region in six mammals, ranging from armadillo to western gorilla. Blue, discontinuous, vertical lines have been used to better visualize the overlap with the human repeat as well as, wherever occurring, the overlap with nonmasked human sequences. Shown in the left flank is the NCBI accession of WGS sequence contigs including the syntenic DNA sequence in each species, followed by the RepeatMasker's annotation of the degenerated repeat element included in the syntenic region. Length of white rhinoceros and armadillo repeats in the figure is not in accordance with the nucleotide content due to the presence of inserts in the syntenic region.

In the same context, out of the 7 common polymorphic sites located in MYEOV long-ORF, all representing single nucleotide substitutions, none induces a nonsense codon. Out of the 6 common polymorphisms included in either MYEOV $5^{\prime}$ UTR or MYEOV intragenic regions, none locates within core splicing signals.

In this context, it is highly plausible that all individuals could be able to encode for the 313-aa peptide.

3.6. MYEOV Peptide(s) May Be Involved Per Se in Promoting the Malignant State. In line with data extracted from COSMIC [48], only 60 MYEOV confirmed somatic mutations were identified in 72 out of 23780 unique samples tested.

The above could signify that the peptide(s) produced by MYEOV may be involved per se, rather than mutated, in promoting the malignant state.

\section{Conclusions}

(i) $M Y E O V$ is a Primate Orphan Gene that acquired, via ORF expansion, a human-protein-specific coding potential. Thus it represents the first cancer-related gene identified to present significantly differing protein-coding potential between human and chimpanzee.

(ii) Should MYEOV role in promoting cancer cell proliferation and metastasis be attributed to MYEOV-313 is a tantalizing question that warrants further investigation, especially in the context that all individuals could be able to encode for this peptide and also because MYEOV-313 may be related per se in promoting tumour propagation and thus could be specifically targeted for. After all, targeting a human-specific peptide would theoretically cause less adverse effects than targeting components of evolutionary conserved signaling cascades. 


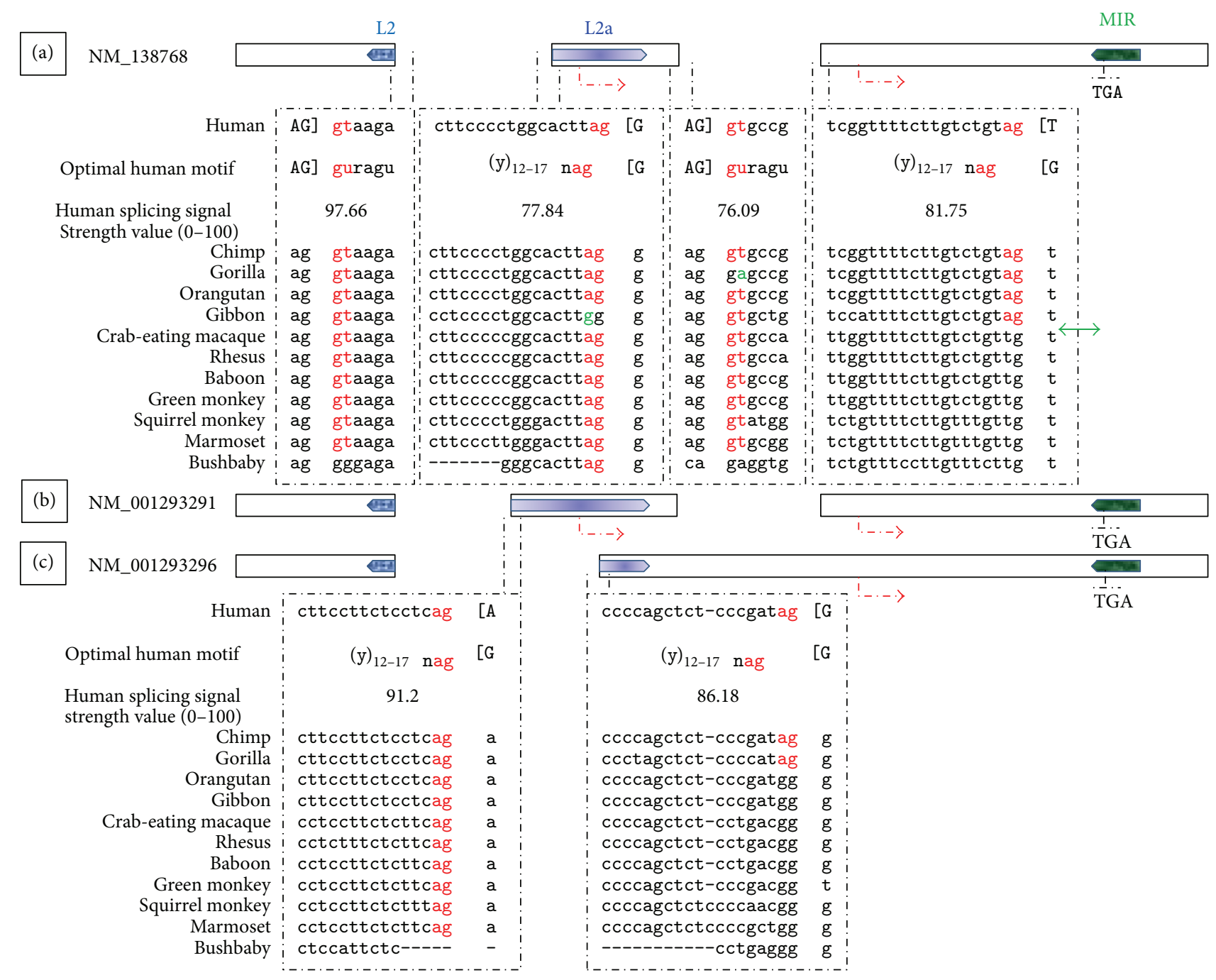

FIGURE 4: MYEOV splicing junctions. Illustrated are three MYEOV alternatively spliced variants (a-c), as described in Figure 1. Shown are the canonical $5^{\prime} / 3^{\prime}$ splice sites that delimit all the putative splicing junctions of MYEOV, as annotated in the Gene database from NCBI. The splice sites are juxtaposed to the optimal core splicing signal motifs; corresponding splicing signal strength values are also shown. Uppercase nucleotides, delimited by brackets, versus lower-case nucleotides correspond to exonic versus intronic content, respectively. Nucleotides exerting the strongest influence on the signal strength appear in red font. Aligned below the human splicing signals appear corresponding nucleotide sequences from MYEOV syntenic region in 11 primates. Shown in green font are nucleotides that deviate from the evolutionary trend, requiring further validation. The double-headed green arrow points to the precious canonical acceptor that arose in hominoids, allowing for MYEOV long-ORF to occur. Alignment gaps correspond to indels located between the aligned blocks in the aligning species.

(iii) It was adaptive evolution coupled with TE exaptation that allowed for the expansion of MYEOV pORF. It is tempting to speculate that it could have all started with the relatively strong core splicing signals provided by the LINE L2a repeat (Figure 4), creating an evolutionary "hotspot" within $M Y E O V$ sequence that was subsequently positively selected for throughout the course of Catarrhini evolution [64].

\section{Data Access}

The sequence data from this study are archived at the Gene database from NCBI (http://www.ncbi.nlm.nih.gov/gene/), the Ensembl Genome Browser Database (http://www.ensembl.org/index.html), and the UCSC Genome Browser Database (http://genome.ucsc.edu/).

\section{Conflict of Interests}

The authors declare that there is no conflict of interests regarding the publication of this paper.

\section{Acknowledgment}

Spyros I. Papamichos gratefully acknowledges Dr. Vassiliki Kotoula for providing fruitful mentoring. 


\section{References}

[1] A. Varki and T. K. Altheide, "Comparing the human and chimpanzee genomes: searching for needles in a haystack," Genome Research, vol. 15, no. 12, pp. 1746-1758, 2005.

[2] A. Varki, "A chimpanzee genome project is a biomedical imperative," Genome Research, vol. 10, no. 8, pp. 1065-1070, 2000.

[3] X. S. Puente, G. Velasco, A. Gutiérrez-Fernández, J. Bertranpetit, M.-C. King, and C. López-Otín, "Comparative analysis of cancer genes in the human and chimpanzee genomes," $B M C$ Genomics, vol. 7, article 15, 2006.

[4] J. W. G. Janssen, M. Cuny, B. Orsetti et al., "MYEOV: a candidate gene for DNA amplification events occurring centromeric to CCND1 in breast cancer," International Journal of Cancer, vol. 102, no. 6, pp. 608-614, 2002.

[5] J. W. G. Janssen, I. Imoto, J. Inoue et al., "MYEOV, a gene at 11q13, is coamplified with CCND1, but epigenetically inactivated in a subset of esophageal squamous cell carcinomas," Journal of Human Genetics, vol. 47, no. 9, pp. 460-464, 2002.

[6] J. W. G. Janssen, J.-W. Vaandrager, T. Heuser et al., "Concurrent activation of a novel putative transforming gene, myeov, and cyclin D1 in a subset of multiple myeloma cell lines with t(11;14)(q13;q32)," Blood, vol. 95, no. 8, pp. 2691-2698, 2000.

[7] G. Lawlor, P. P. Doran, P. MacMathuna, and D. W. Murray, "MYEOV (myeloma overexpressed gene) drives colon cancer cell migration and is regulated by PGE2," Journal of Experimental and Clinical Cancer Research, vol. 29, article 81, 2010.

[8] J. Leyden, D. Murray, A. Moss et al., "Netl and Myeov: computationally identified mediators of gastric cancer," British Journal of Cancer, vol. 94, no. 8, pp. 1204-1212, 2006.

[9] J. Moreaux, D. Hose, A. Bonnefond et al., "MYEOV is a prognostic factor in multiple myeloma," Experimental Hematology, vol. 38, no. 12, pp. 1189-1198.e3, 2010.

[10] A. C. Moss, G. Lawlor, D. Murray et al., "ETV4 and Myeov knockdown impairs colon cancer cell line proliferation and invasion," Biochemical and Biophysical Research Communications, vol. 345, no. 1, pp. 216-221, 2006.

[11] J. Takita, Y. Chen, J. Okubo et al., "Aberrations of NEGR1 on 1p31 and MYEOV on 11q13 in neuroblastoma," Cancer Science, vol. 102, no. 9, pp. 1645-1650, 2011.

[12] R. A. De Almeida, T. Heuser, R. Blaschke, C. R. Bartram, and J. W. G. Janssen, "Control of MYEOV protein synthesis by upstream open reading frames," The Journal of Biological Chemistry, vol. 281, no. 2, pp. 695-704, 2006.

[13] M. Kozak, "Downstream secondary structure facilitates recognition of initiator codons by eukaryotic ribosomes," Proceedings of the National Academy of Sciences of the United States of America, vol. 87, no. 21, pp. 8301-8305, 1990.

[14] M. Kozak, "Pushing the limits of the scanning mechanism for initiation of translation," Gene, vol. 299, no. 1-2, pp. 1-34, 2002.

[15] M. Kozak, "Regulation of translation via mRNA structure in prokaryotes and eukaryotes," Gene, vol. 361, no. 1-2, pp. 13-37, 2005.

[16] C. Xie, Y. E. Zhang, J.-Y. Chen et al., "Hominoid-specific de novo protein-coding genes originating from long non-coding RNAs," PLoS Genetics, vol. 8, no. 9, Article ID e1002942, 2012.

[17] D. G. Knowles and A. McLysaght, "Recent de novo origin of human protein-coding genes," Genome Research, vol. 19, no. 10, pp. 1752-1759, 2009.
[18] M. Long, E. Betrán, K. Thornton, and W. Wang, “The origin of new genes: glimpses from the young and old," Nature Reviews Genetics, vol. 4, no. 11, pp. 865-875, 2003.

[19] R. Cordaux and M. A. Batzer, "The impact of retrotransposons on human genome evolution," Nature Reviews Genetics, vol. 10, no. 10, pp. 691-703, 2009.

[20] H. H. Kazazian Jr., "Mobile elements: drivers of genome evolution," Science, vol. 303, no. 5664, pp. 1626-1632, 2004.

[21] R. J. Britten, "Mobile elements inserted in the distant past have taken on important functions," Gene, vol. 205, no. 1-2, pp. 177182, 1997.

[22] M. Krull, M. Petrusma, W. Makalowski, J. Brosius, and J. Schmitz, "Functional persistence of exonized mammalian-wide interspersed repeat elements (MIRs)," Genome Research, vol. 17, no. 8, pp. 1139-1145, 2007.

[23] M. Wu, L. Li, and Z. Sun, "Transposable element fragments in protein-coding regions and their contributions to human functional proteins," Gene, vol. 401, no. 1-2, pp. 165-171, 2007.

[24] A. F. Smit, "Interspersed repeats and other mementos of transposable elements in mammalian genomes," Current Opinion in Genetics and Development, vol. 9, no. 6, pp. 657-663, 1999.

[25] J. Brosius and S. J. Gould, “On 'genomenclature': a comprehensive (and respectful) taxonomy for pseudogenes and other 'junk DNA"' Proceedings of the National Academy of Sciences of the United States of America, vol. 89, no. 22, pp. 10706-10710, 1992.

[26] S.-T. Chen, H.-C. Cheng, D. A. Barbash, and H.-P. Yang, "Evolution of hydra, a recently evolved testis-expressed gene with nine alternative first exons in Drosophila melanogaster," PLoS Genetics, vol. 3, no. 7, article e107, 2007.

[27] H. Kaessmann, "Origins, evolution, and phenotypic impact of new genes," Genome Research, vol. 20, no. 10, pp. 1313-1326, 2010.

[28] Q. Zhou and W. Wang, "On the origin and evolution of new genes-a genomic and experimental perspective," Journal of Genetics and Genomics, vol. 35, no. 11, pp. 639-648, 2008.

[29] C. Y. Li, Y. Zhang, Z. Wang et al., "A human-specific de novo protein-coding gene associated with human brain functions," PLoS Computational Biology, vol. 6, no. 3, Article ID e1000734, 2010.

[30] M. Blanchette, W. J. Kent, C. Riemer et al., "Aligning multiple genomic sequences with the threaded blockset aligner," Genome Research, vol. 14, no. 4, pp. 708-715, 2004.

[31] D. Karolchik, G. P. Barber, J. Casper et al., "The UCSC Genome Browser database: 2014 update," Nucleic Acids Research, vol. 42, no. 1, pp. D764-D770, 2014.

[32] K. S. Pollard, M. J. Hubisz, K. R. Rosenbloom, and A. Siepel, "Detection of nonneutral substitution rates on mammalian phylogenies," Genome Research, vol. 20, no. 1, pp. 110-121, 2010.

[33] S. Schwartz, W. J. Kent, A. Smit et al., "Human-mouse alignments with BLASTZ," Genome Research, vol. 13, no. 1, pp. 103107,2003

[34] S. F. Altschul, W. Gish, W. Miller, E. W. Myers, and D. J. Lipman, "Basic local alignment search tool," Journal of Molecular Biology, vol. 215, no. 3, pp. 403-410, 1990.

[35] D.-D. Wu, D. M. Irwin, and Y.-P. Zhang, "De novo origin of human protein-coding genes," PLoS Genetics, vol. 7, no. 11, Article ID e1002379, 2011.

[36] R. M. Waterhouse, F. Tegenfeldt, J. Li, E. M. Zdobnov, and E. V. Kriventseva, "OrthoDB: a hierarchical catalog of animal, fungal and bacterial orthologs," Nucleic Acids Research, vol. 41, no. 1, pp. D358-D365, 2013. 
[37] W. Li, A. Cowley, M. Uludag et al., “The EMBL-EBI bioinformatics web and programmatic tools framework," Nucleic Acids Research, vol. 43, no. 1, pp. W580-W584, 2015.

[38] L. D. Hurst, "The $\mathrm{Ka} / \mathrm{Ks}$ ratio: diagnosing the form of sequence evolution," Trends in Genetics, vol. 18, no. 9, pp. 486-487, 2002.

[39] K. Tamura, G. Stecher, D. Peterson, A. Filipski, and S. Kumar, "MEGA6: molecular evolutionary genetics analysis version 6.0," Molecular Biology and Evolution, vol. 30, no. 12, pp. 2725-2729, 2013.

[40] K. Finstermeier, D. Zinner, M. Brameier et al., "A mitogenomic phylogeny of living primates," PLoS ONE, vol. 8, no. 7, Article ID e69504, 2013.

[41] A. Smit, R. Hubley, and P. Green, "RepeatMasker Open-3.0," 1996-2010, http://www.repeatmasker.org/.

[42] G. Tóth, G. Deák, E. Barta, and G. B. Kiss, "PLOTREP: a web tool for defragmentation and visual analysis of dispersed genomic repeats," Nucleic Acids Research, vol. 34, supplement 2, pp. W708-W713, 2006.

[43] A. Levy, N. Sela, and G. Ast, "TranspoGene and microTranspoGene: transposed elements influence on the transcriptome of seven vertebrates and invertebrates," Nucleic Acids Research, vol. 36, no. 1, pp. D47-D52, 2008.

[44] A. Morgulis, E. M. Gertz, A. A. Schäffer, and R. Agarwala, "A fast and symmetric DUST implementation to mask low-complexity DNA sequences," Journal of Computational Biology, vol. 13, no. 5, pp. 1028-1040, 2006.

[45] S. E. Calvo, D. J. Pagliarini, and V. K. Mootha, "Upstream open reading frames cause widespread reduction of protein expression and are polymorphic among humans," Proceedings of the National Academy of Sciences of the United States of America, vol. 106, no. 18, pp. 7507-7512, 2009.

[46] F.-O. Desmet, D. Hamroun, M. Lalande, G. Collod-Bëroud, M. Claustres, and C. Béroud, "Human Splicing Finder: an online bioinformatics tool to predict splicing signals," Nucleic Acids Research, vol. 37, no. 9, article e67, 2009.

[47] S. I. Papamichos, "An Alu exonization event allowing for the generation of a novel OCT4 isoform," Gene, vol. 512, no. 1, pp. 175-177, 2013.

[48] S. A. Forbes, G. Tang, N. Bindal et al., "COSMIC (the Catalogue of Somatic Mutations in Cancer): a resource to investigate acquired mutations in human cancer," Nucleic Acids Research, vol. 38, supplement 1, pp. D652-D657, 2010.

[49] M. Clamp, B. Fry, M. Kamal et al., "Distinguishing proteincoding and noncoding genes in the human genome," Proceedings of the National Academy of Sciences of the United States of America, vol. 104, no. 49, pp. 19428-19433, 2007.

[50] A. Siepel, "Darwinian alchemy: human genes from noncoding DNA," Genome Research, vol. 19, no. 10, pp. 1693-1695, 2009.

[51] V. Curwen, E. Eyras, T. D. Andrews et al., "The Ensembl automatic gene annotation system," Genome Research, vol. 14, no. 5, pp. 942-950, 2004.

[52] M. Nei, "Selectionism and neutralism in molecular evolution," Molecular Biology and Evolution, vol. 22, no. 12, pp. 2318-2342, 2005.

[53] M. Nei, Y. Suzuki, and M. Nozawa, "The neutral theory of molecular evolution in the genomic era," Annual Review of Genomics and Human Genetics, vol. 11, pp. 265-289, 2010.

[54] J. M. Akey, "Constructing genomic maps of positive selection in humans: where do we go from here?" Genome Research, vol. 19, no. 5, pp. 711-722, 2009.
[55] G. Bejerano, C. B. Lowe, N. Ahituv et al., "A distal enhancer and an ultraconserved exon are derived from a novel retroposon," Nature, vol. 441, no. 7089, pp. 87-90, 2006.

[56] N. Lovšin, F. Gubenšek, and D. Kordiš, "Evolutionary dynamics in a novel L2 clade of non-LTR retrotransposons in Deuterostomia," Molecular Biology and Evolution, vol. 18, no. 12, pp. 22132224, 2001.

[57] E. S. Lander, L. M. Linton, B. Birren et al., "Initial sequencing and analysis of the human genome," Nature, vol. 409, no. 6822, pp. 860-921, 2001.

[58] J. Jurka, V. V. Kapitonov, A. Pavlicek, P. Klonowski, O. Kohany, and J. Walichiewicz, "Repbase Update, a database of eukaryotic repetitive elements," Cytogenetic and Genome Research, vol. 110, no. 1-4, pp. 462-467, 2005.

[59] P. L. Deininger and M. A. Batzer, "Mammalian retroelements," Genome Research, vol. 12, no. 10, pp. 1455-1465, 2002.

[60] C. W. Cunningham, K. E. Omland, and T. H. Oakley, "Reconstructing ancestral character states: a critical reappraisal," Trends in Ecology and Evolution, vol. 13, no. 9, pp. 361-366, 1998.

[61] M. Blanchette, E. D. Green, W. Miller, and D. Haussler, "Reconstructing large regions of an ancestral mammalian genome in silico," Genome Research, vol. 14, no. 12, pp. 2412-2423, 2004.

[62] G. Li, J. Ma, and L. Zhang, "Greedy selection of species for ancestral state reconstruction on phylogenies: elimination is better than insertion," PLoS ONE, vol. 5, no. 2, Article ID e8985, 2010.

[63] Z. Wang and C. B. Burge, "Splicing regulation: from a parts list of regulatory elements to an integrated splicing code," RNA, vol. 14, no. 5, pp. 802-813, 2008.

[64] Y. Xing and C. Lee, "Evidence of functional selection pressure for alternative splicing events that accelerate evolution of protein subsequences," Proceedings of the National Academy of Sciences of the United States of America, vol. 102, no. 38, pp. 13526-13531, 2005. 

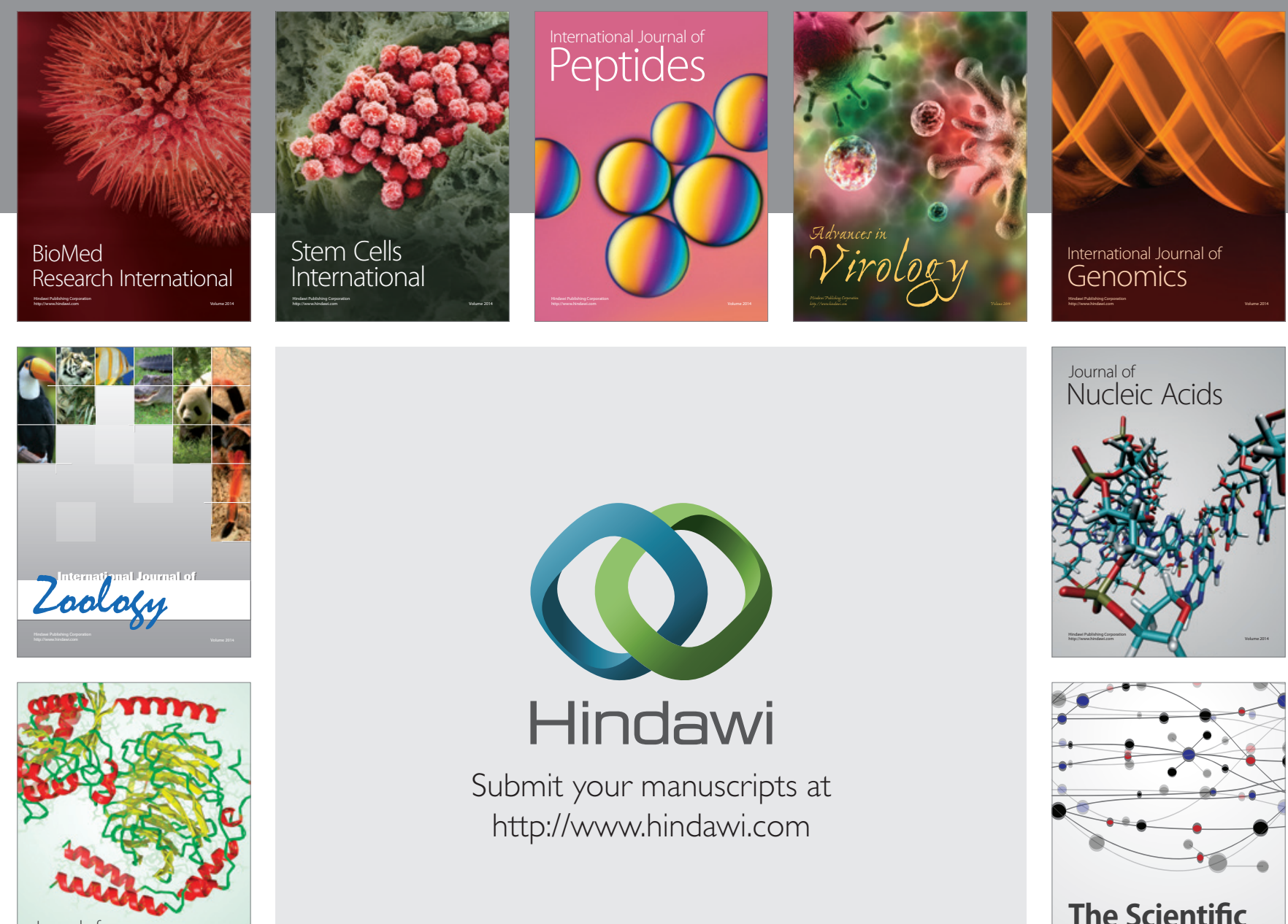

Submit your manuscripts at

http://www.hindawi.com

Journal of
Signal Transduction
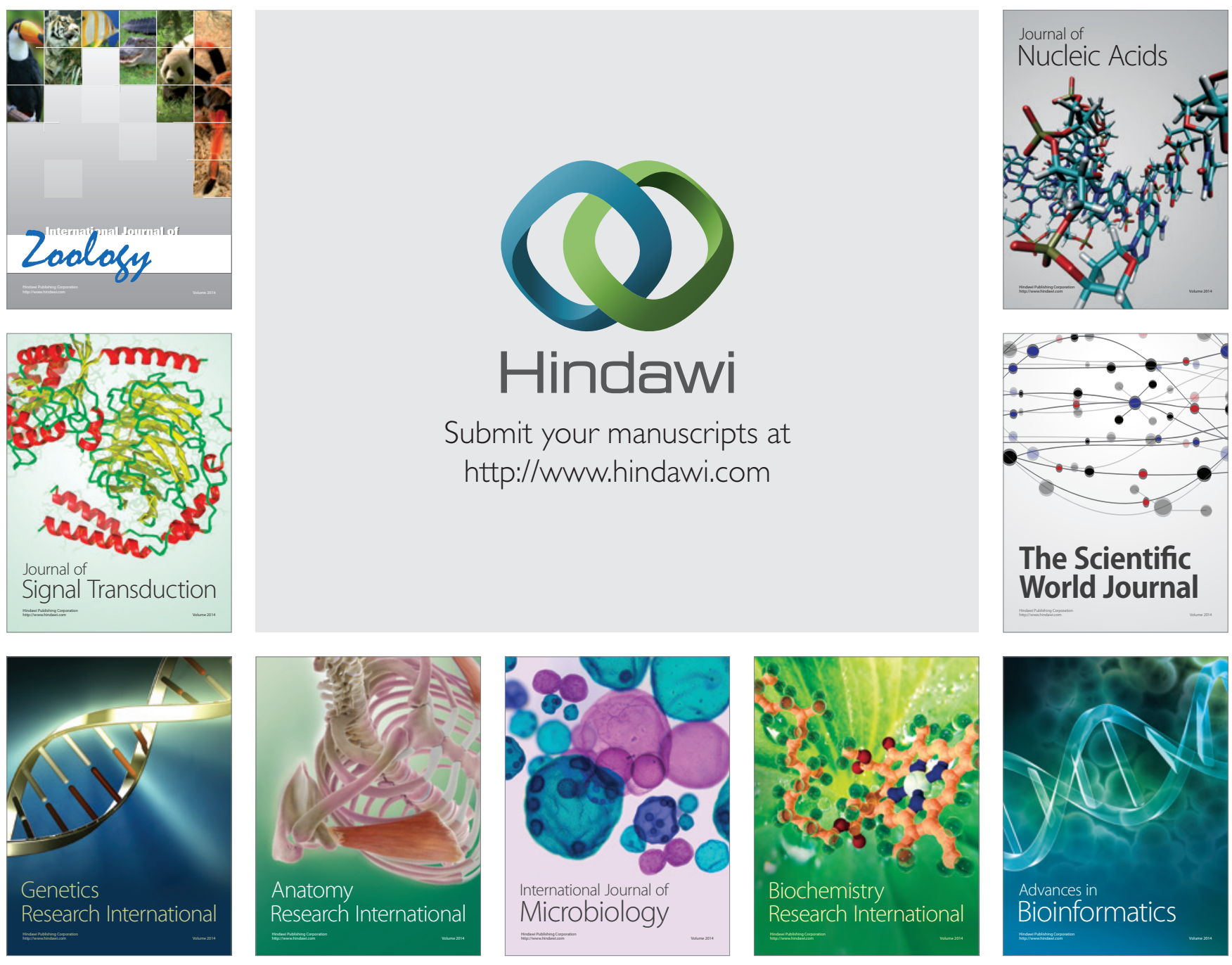

The Scientific World Journal
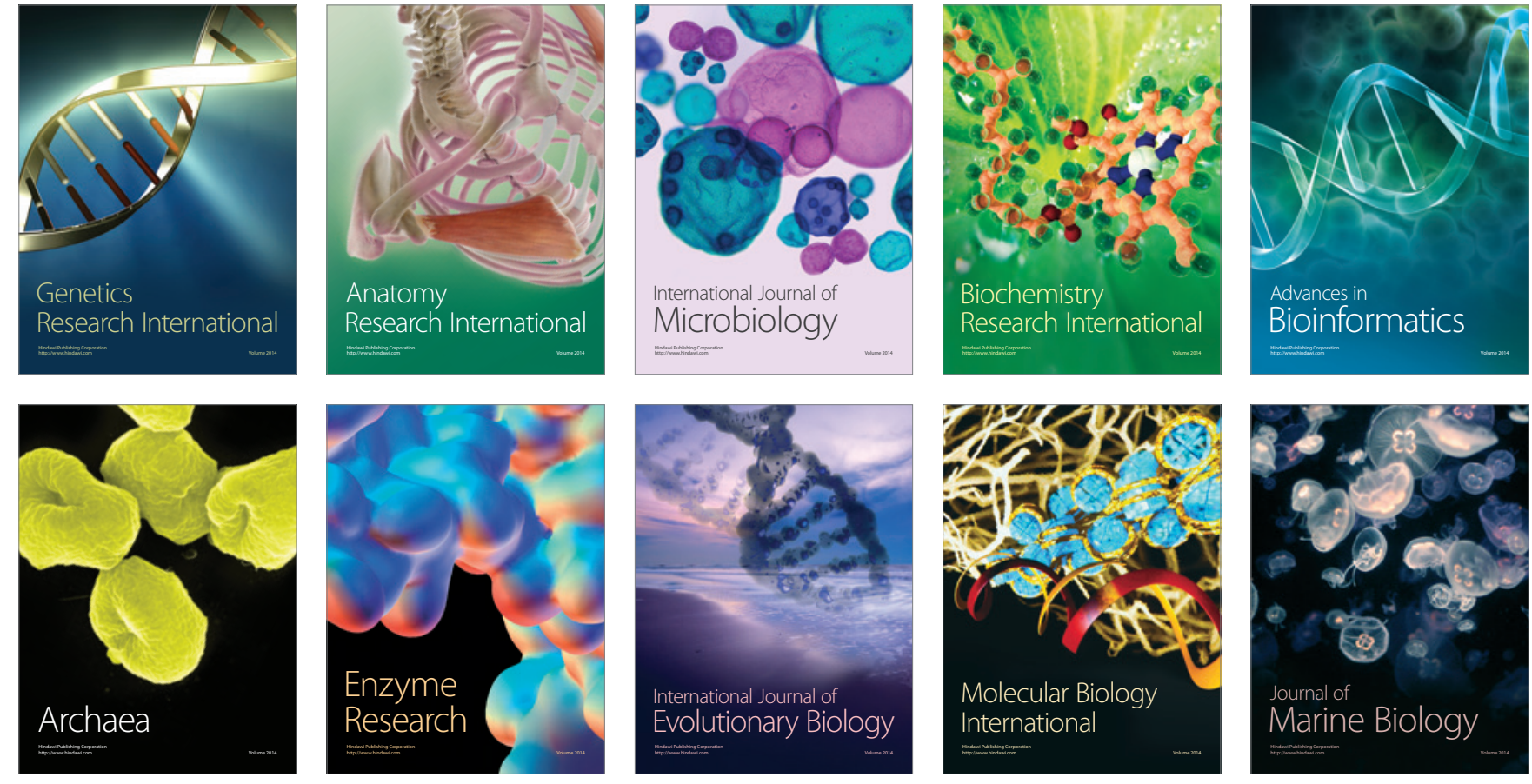